\title{
INVESTIGATION OF SURFACE FUNCTIONALIZATION AND COATINGS FOR BIOMEDICAL APPLICATIONS BY ZETA POTENTIAL AND ADSORPTION MEASUREMENTS ON SOLID SURFACES
}

\author{
SILVIA SPRIANO, SARA FERRARIS, MARTINA CAZZOLA \& VERONICA PERETTI \\ Department of Applied Science and Technology, Politecnico di Torino, Italy
}

\begin{abstract}
Even though zeta potential titration is widely employed for investigation of colloidal suspensions, it is still poorly applied to bulk solid surfaces. The measurement can be applied to surfaces in contact with water-based media, and it gives information about the isoelectric point of the surfaces, surface charge in function of $\mathrm{pH}$, functional groups exposed on the surface, and adsorption/desorption processes at the solid-liquid interface. Zeta potential titration vs $\mathrm{pH}$ is useful for applications such as membranes, filters, biomaterials, semiconductors, textile fibres, cosmetic, detergents and minerals. In the present research, zeta potential of Ti6Al4V was measured using electro kinetic measurements (SurPASS, Anton Paar) after surface treatments, functionalization or coating. Zeta potential was measured, in function of $\mathrm{pH}$, in a $0.001 \mathrm{M} \mathrm{KCl}$ electrolyte solution; varying the solution of $\mathrm{pH}$ by addition of $0.05 \mathrm{M}$ $\mathrm{HCl}$ or $\mathrm{NaOH}$ through the instrument's automatic titration unit. Moreover, the effects of adsorption/desorption of ions and proteins after soaking in Simulated Body Fluid (SBF) and albumin (BSA) were tested. The tested Ti6Al4V samples were: 1) chemically treated samples finalized to better osseointegration through nanoscale surface topography, high hydroxylation degree and, eventually, an antibacterial action through silver nanoparticles; 2) surface functionalized samples with biomolecules for antibacterial and/or anti-inflammatory purposes (gallic acid as an example); 3) a coating of titanium boride finalized for better fretting, corrosion and wear resistance in artificial joints. The data will be compared and discussed. The technique is useful in order to compare exposed specific functional groups on the surface, and to measure the zeta potential at physiologic $\mathrm{pH}$ and the effects of ions adsorption on the surface charge, which is relevant for bioactive and antibacterial behaviour, as well as of protein adsorption which is also relevant for lubrication and wear in artificial joints.
\end{abstract}

Keywords: biomaterials, zeta potential, surfaces, adsorption, bioactivity, functionalization, lubrication.

\section{INTRODUCTION}

Surface properties are a critical issue for biomaterials in their working environment, as evident in the case of the surface of implants in contact with the biological fluids or tissues. The main surface properties affecting the biological response of biomaterials are roughness, chemical composition, surface charge, wettability and hydroxylation degree. Among them, surface charge is a poorly explored property, especially in the case of bulk materials.

Solid surfaces of biomaterials can show several functional groups (e.g. hydroxyl, carboxyl or amine groups) or no specific chemical functionalities. Upon contact with water based media, a surface charge is developed at the interface with the liquid medium by means of acid-base reactions of the surface (e.g. dissociation of acidic hydroxyl groups, protonation of amine groups or basic hydroxyl groups and consequent development of respectively negative and positive charges) or by means of replacement of the adsorbed water molecules by ions $\left(\mathrm{OH}^{-}, \mathrm{H}_{3} \mathrm{O}^{+}\right)$on the surfaces with no specific functional groups exposed [1].

The charge distribution at the surface-liquid interface induces a consequent counterions distribution in the liquid. According to the Electrochemical Double Layer (EDL) model, a 
stationary immobile layer (stationary layer) and a diffuse mobile layer (diffuse layer) of counterions are developed in order to compensate the surface charge. The surface potential decays increasing the distance from the surface. The zeta potential is defined as the potential at the outside of the stationary layer [1]. Usually, electrophoretic mobility is considered for determination of zeta potential of the colloidal suspensions while streaming potential/current is used for characterization of charging behaviour of the bulk surfaces. The flow of a fluid on the surface of a material causes the motion of the counterions that compensate the surface charge in the direction of the flow. The charge separation causes an electrical potential difference called streaming potential. While zeta potential is a widely studied property in the case of colloidal suspensions (for the investigation of their stability), it is still poorly studied in the case of the bulk materials. In this case, zeta potential describes surface charging behaviour in contact with water based media and gives information about the functional groups exposed at the solid-liquid interface, the interaction of the surface with ions or compounds dissolved in the solution and on adsorption/desorption processes [1].

Information of interest for various application fields (e.g. membranes and filters, biomaterials, semiconductors, fibres, cosmetic and detergents, polymers and minerals) and on different materials (oxides, metals and polymers) can be obtained from the zeta potential measurements on bulk materials. In the present research, Ti6Al4V alloy was investigated after different surface treatments, functionalization or coating protocols of interest for application as orthopaedic or dental implants.

\section{EXPERIMENTAL SECTION}

The processes of surface treatment, functionalization and coating on Ti6Al4V alloy used for obtaining the samples analysed in this research work were already described elsewhere: the main issues are here summarized.

Case 1 concerns a chemical treatment finalized to better response to hard tissues (osseointegration) through nano-scaled surface topography, high hydroxylation degree and, eventually, an antibacterial action through silver nanoparticles enrichment. Plane disks (2 $\mathrm{mm}$ thickness and $10 \mathrm{~mm}$ diameter) were obtained from cylindrical bars of Ti6Al4V alloy (ASTM B348, Gr5, Titanium Consulting and Trading) by means of an automatic cutting machine (Struers Accutom 5) equipped with an alumina blade (356 CA). The samples were then polished with $\mathrm{SiC}$ abrasive papers (up to 4000 grit), washed in an ultrasonic bath in acetone (one time for $5 \mathrm{~min}$ ) and in ultrapure water (two times for $10 \mathrm{~min}$ ). The surface of the samples was treated, according to a patented chemical treatment [2]. The treatment foresees a first surface etching in hydrofluoric acid in order to remove the native oxide layer and a subsequent controlled oxidation in hydrogen peroxide in order to obtain the nanotextured titanium oxide. The hydrogen peroxide can be eventually enriched with silver nitrate $\left(\mathrm{AgNO}_{3}\right.$, Silver Nitrate PA-ACS-ISO 131459,1611, Panreac) in order to get silver nanoparticles embedded within the surface oxide layer [3]. Acid gallic and polyvinyl alcohol were added to the solution of silver nitrate as reducing/stabilizing agents, in order to control silver nanoparticles size and distribution. The samples treated without silver ions and reducing/stabilizing agents will be called "Ti-Treat" in the text, while the samples treated with the addition of silver ions and reducing/stabilizing agents will be called "TiTreat-Ag".

Case 2 concerns surface functionalization with biomolecules for antibacterial and/or anti-inflammatory purposes [4]. Plane disks of Ti6A14V alloy were treated as in Case 1 (without the addition of silver nitrate and reducing/stabilizing agents), activated by UV irradiation and then functionalized by soaking in a solution of $1 \mathrm{mg} / \mathrm{ml}$ gallic acid $(3,4,5$ - 
Trihydroxybenzoic acid, GA, 97.5-102.5\% titration, G7384, Sigma-Aldrich) in Simulated Body Fluid (SBF). Gallic acid was selected as a simple model molecule for polyphenols which are of interest for their antioxidant, neuroprotective and antitumoral properties. The SBF solution was prepared according to the protocol described by Kokubo [5]. Each sample, was soaked in $5 \mathrm{ml}$ of the solution, in a holder coated with an aluminium foil in order to avoid UV degradation of the biomolecule, for $8 \mathrm{~h}$ at $37^{\circ} \mathrm{C}$. After incubation, the samples were washed two times with ultrapure water, dried at room temperature and preserved in the dark. The samples functionalized with gallic acid will be called "Ti-TreatFunc" in the text.

Case 3 concerns a coating of titanium boride on Ti6Al4V alloy finalized to better fretting, corrosion and wear resistance in artificial joints. Samples $10 \mathrm{~mm}$ in diameter and $2 \mathrm{~mm}$ thick were manually polished using SiC grinding papers from 120 to 4000 grits. A boriding powder mixture composed of $50 \mathrm{wt} \% \mathrm{~B}, 15 \mathrm{wt} \% \quad \mathrm{Na}_{2} \mathrm{~B}_{4} \mathrm{O}_{7}$ and $35 \mathrm{wt} \% \mathrm{C}$ was prepared by milling the powders for about $6 \mathrm{~h}$ in a $\mathrm{WC}$ ball milling in air. The mixture was packed in sealed graphite crucibles in an Ar filled gloves box ensuring a sufficiently thick pack $(2.5 \mathrm{~cm})$ all-around the samples. The thermal treatment diffusion processes were performed by following the protocol of B. Sarman et al. [6]. The pack was heated (rate: $10^{\circ} \mathrm{C} / \mathrm{min}$ ) up to $800 \mathrm{C}^{\circ}$ and hold for $3.5 \mathrm{~h}$ in a tubular furnace in $\mathrm{Ar}$ atmosphere and then cooled at room temperature within the furnace. The samples coated by titanium boride will be called "Ti-Coat" in the text. Some Ti-Coat samples were soaked for $1 \mathrm{~h}$ in a water solution of Bovine Serum Albumin (BSA) of $20 \mathrm{mg} / \mathrm{ml}$ (samples called Ti-Coat_ads) and when tested by zeta potential titration. As reference, some alumina samples (Expert System Solutions S.R.L.) were soaked in the same conditions (samples called Alumina_ads in the text).

An electro kinetic analyser (SurPASS, Anton Paar) has been employed for zeta potential titration vs $\mathrm{pH}$. The surface zeta potential was determined in function of $\mathrm{pH}$ in a $0.001 \mathrm{M}$ $\mathrm{KCl}$ electrolyte solution varying the solution $\mathrm{pH}$ by addition of $0.05 \mathrm{M} \mathrm{HCl}$ or $0.05 \mathrm{M} \mathrm{NaOH}$ through the instrument automatic titration unit. The acid and alkaline sides of the curve were obtained in two different steps, usually firstly testing the acid one.

\section{RESULTS AND DISCUSSION}

Ti6Al4V alloy was investigated after different surface treatments: chemical treatments (samples called Ti-Treat and Ti-Treat-Ag - case 1), functionalization with gallic acid (samples called Ti-Treat-Func - case 2) and coating by titanium boride (samples called TiCoat - case 3). All these materials are of interest for application as orthopaedic or dental implants. The data obtained by using zeta potential titration vs $\mathrm{pH}$ on these materials are here presented. This characterization technique is useful in order to evaluate and compare the isoelectric point, the zeta potential at physiologic $\mathrm{pH}$, the eventual exposition on the surfaces of specific functional groups, the eventual adsorption and/or desorption phenomena in function of $\mathrm{pH}$ and the effects of ions/proteins adsorption, occurred during soaking before the test, on the surface charge at different $\mathrm{pH}$. The adsorption/release of ions after soaking in SBF (cases 1 and 2) is relevant for bioactive and antibacterial behaviour, while protein adsorption is also relevant for lubrication and wear during friction in artificial joints (case 3).

\subsection{Surface treatments on the Ti6Al4V alloy}

Osseointegration is a pre-requisite for successful implant rehabilitation: bone implant surfaces should promote formation of sound bone around the implant body. Most commercially available dental and orthopaedic implant systems show micro-rough surface 
patterns on titanium or titanium alloys: rough surfaces promote cell commitment to the osteoblastic lineage and support higher expression of phenotype-specific markers, but the characteristics of the ideal surface able to maximise bone growth are still unknown and the question is open to research [7]. As a consequence, several treatment protocols are still being investigated, to better define the degree of roughness, and the optimal physicochemical features to promote colonisation, cell differentiation and, ultimately, bone formation on the titanium surface. Nano-scaled roughness is attracting great interest because it can stimulate cells without increasing bacterial adhesion. The surface treatment here investigated (Ti-Treat samples- see the Experimental Section for a description of the process) develops a peculiar surface oxide layer through a chemical treatment. It differs from the native titanium oxide for several features: higher thickness, nano-texture (porosity on the nanoscale), inorganic bioactivity (in vitro apatite precipitation), higher wettability, higher corrosion resistance, active sites for functionalization [8]. Inorganic bioactivity and spontaneous hydroxyapatite nucleation on the titanium surface in contact with physiologic fluids is quite interesting in order to get the formation of the mineral part of the bone [9].

A variation of the chemical surface treatment includes the addition of silver ions and reducing/stabilizing agents in order to obtain the nucleation of silver nanoparticles embedded within the surface oxide layer (Ti-Treat-Ag). This material is of interest both for effective osseointegration and antibacterial action mainly because of silver ion release [10].

A comprehensive characterization of these surfaces is reported in the papers abovementioned. Specific surface characteristics are here investigated: isoelectric point, zeta potential at physiologic $\mathrm{pH}(\mathrm{pH}=7.4)$ and adsorption/desorption effects $\mathrm{vs} \mathrm{pH}$.

The following samples were analysed (Fig. 1): untreated Ti6Al4V alloy and untreated metal silver samples (as reference materials), Ti6Al4V chemically treated samples without (Ti-Treat) or with (Ti-Treat-Ag) the addition of silver ions and reducing/stabilizing agents during the process.

The untreated Ti6Al4V shows the isoelectric point at $\mathrm{pH}=4.7$, in accordance with the data reported in literature [11], while the surface chemical treatments induce a shift of the isoelectric point to more acidic values (close to $\mathrm{pH}=2$ ) irrespective of the presence or not of silver nanoparticles on the surface. Untreated bulk metal silver shows the isoelectric point at $\mathrm{pH}$ no far from 2 thus the presence of silver nanoparticles is expected to have no significant effect on the isoelectric point of the treated surface. The definition of isoelectric point is the $\mathrm{pH}$ value where the electro kinetic charge density and zeta potential are zero; the zeta potential reverses its sign at this $\mathrm{pH}$ [1]. Surfaces with isoelectric point in the acid range can be defined as acidic and a shift of the isoelectric point towards lower $\mathrm{pH}$ values is related to a high surface density of acidic functional groups. A first information from the zeta potential measurement here reported is that the explored chemically surface treatments increase the presence of one or more acidic groups, while the untreated Ti6Al4V alloy do not show a consistent amount of specific functional groups.

The zeta potential at physiologic $\mathrm{pH}(\mathrm{pH}=7.4)$ is highly negative in the case of the untreated Ti6Al4V alloy $(-65 \mathrm{mV})$ while it shifts to less negative values $(-40 \mathrm{mV})$ in the case of both the chemically treated samples (Ti-Treat and Ti-Treat-Ag) without significant effects due to the presence of silver nanoparticles. This difference is related to a different zeta potential trend vs $\mathrm{pH}$ on the chemically treated samples with respect to the untreated reference (Ti6Al4V alloy and pure silver). The trend of the titration curve of the untreated alloy is straightforward much more negative values almost within the overall tested $\mathrm{pH}$ range. This corresponds to a progressive replacement of the adsorbed water molecules by water ions ( $\mathrm{OH}$ groups in the case of the alkaline range). Differently, a plateau in the basic region (from $\mathrm{pH} 5.5$ to $\mathrm{pH} 8.5$ ) appears in the case of the chemically treated samples. A 
plateau of the titration curve in the alkaline range is attributable to the presence on the surface of the material of one specific homogeneous functional group with acidic behaviour [1]. This group is completely dissociated (deprotonation/ionization is complete) at the $\mathrm{pH}$ value where the plateau starts and thus a further increase of $\mathrm{pH}$ in the alkaline range does not involve any change in the surface charge of the surface. A comprehensive evaluation of the experimental data available for these materials (such as FTIR and XPS analyses reported in [8]) allows to identify this functional group as $\mathrm{OH}$. The position of the plateau of the zeta potential on the vertical and horizontal axes of the graph is related to the density and stronger/weaker acidic character of the functional group exposed on the surface. Some papers in literature [12] report that strong acids are deprotonated at $\mathrm{pH}$ around 2-3, while the carboxylic group is completely dissociated at $\mathrm{pH}$ around $4-5$ and alcoholic group gets a plateau at a $\mathrm{pH}$ value only higher than 8 . Thus, further information we get from the zeta potential measurements is that the chemically treated Ti6Al4V surfaces mainly expose one type of acidic functional group, which has an acidic character, a little bit weaker than organic acids, and it is completely ionized at the physiologic pH. Moreover, both XPS, FTIR [10] and zeta potential data agree that the amount of the exposed hydroxyl group is quite similar on the Ti-Treat and Ti-Treat-Ag samples. XPS data show that silver is present mainly as neutral metal nanoparticles on the surface of the Ti-Treat-Ag, even if a small contribution from silver ions is present. It cannot be excluded that the surface charge effects due to silver positive ions and/or nanoparticles, as reported in [13], could be partially masked by the presence of negative $\mathrm{OH}$ groups on the Titanium surface.

Some fluctuations are present in the curves of the chemically treated samples before the plateau: two changes of the slope of the curve are observable at $\mathrm{pH} 3$ and 4.5-5, while around $\mathrm{pH} 8.5$ the curves have a reversal trend that is towards positive charge in the case of the Ti-Treat-Ag and untreated alloy, while it is towards negative charge in the case of the Ti-Treat sample. At the moment, we are not able to link these signals to specific surface features even if the presence of some $\mathrm{OH}$ groups respectively stronger and weaker than the predominant type, in different amount on the different surfaces, can be assumed. The presence of several $\mathrm{OH}$ groups with different acidic behaviour on the same surface giving a cascade of plateaux at different $\mathrm{pH}$ values is reported in literature in the case of polymers [12].

Concerning the information that can be derived from the zeta potential measurements, the presence of a plateau in the zeta potential curve can be associated to a hydrophilic behaviour [1], precisely because a functional group preferentially exposed on the surface can have a specific interaction with the water molecules if organic contaminations are absent [14]. The zeta potential measurements are almost independent from the roughness of the surface and strictly linked to the surface chemistry: on the other side, wettability measurements by contact angle goniometer are strongly affected by roughness of the sample and it is not easy to separate chemical and topographical effects.

Describing the biological and clinical aspects, generation of a less negative surface charge on the surface can facilitate adsorption of cellular adhesion proteins, which are negatively charged, such as fibronectin [12]. Moreover, the presence of $\mathrm{OH}$ groups on the surface is related to adsorption of calcium and phosphate ions and finally to the bioactive behaviour and precipitation of hydroxyapatite, as it will be described in the following.

In order to investigate the bioactive behaviour (apatite precipitation) of the chemically treated surfaces, the titration curves of zeta potential (in the basic range) of some samples after soaking in SBF are reported in the inserted chart of Fig. 1. The analysed samples are Ti-Treat and Ti-Treat-Ag after 14 days of soaking in SBF (called Ti-Treat_14 dd SBF and Ti-Treat-Ag_14 dd soaking SBF). 


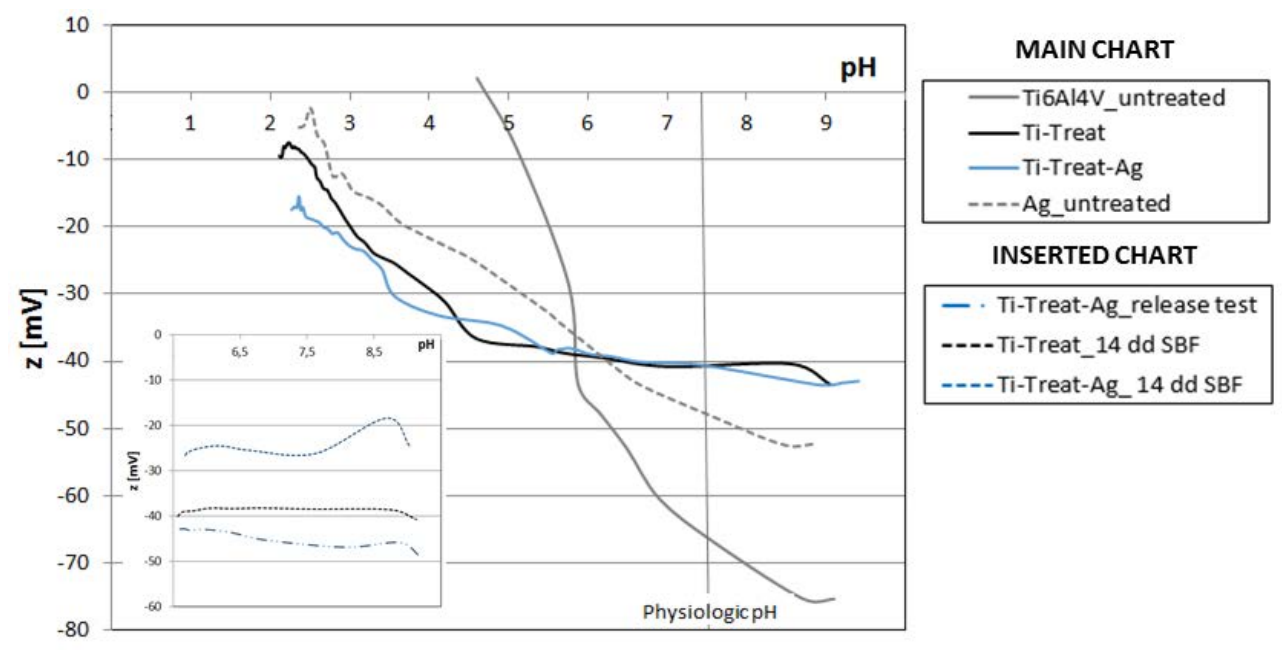

Figure 1: Zeta potential titration vs $\mathrm{pH}$ of the following samples: Ti6Al4V untreated alloy, Ti-Treat, Ti-Treat-Ag, Ag untreated metal (main chart), Ti-Treat-Ag after the silver release test, Ti-Treat after15 days of soaking in SBF, Ti-TreatAg after15 days of soaking in SBF.

The titration curve of the Ti-Treat $14 \mathrm{dd}$ SBF sample in the alkaline range is not so much different from what observed on the Ti-Treat sample. This is interesting because SEM observations as well as XPS and EDS data [15] show on this sample the presence of calcium phosphate crystals with a $\mathrm{Ca} / \mathrm{P}$ ratio of about 1.1 (different from the stoichiometric value of hydroxyapatite). The crystals do not cover all the surface and the substrate with adsorbed ions is somewhere exposed. In this case, the titration curve in the alkaline range is useful in order to evaluate the kinetic of bioactive behaviour. As it will be explained in the following, stable hydroxyapatite shows a much more negative charge because of a preferential surface exposition of phosphate groups [16]. The small difference of this titration curve with respect to the un-soaked sample suggests, in agreement with the EDS/XPS data, that on this sample the precipitation of hydroxyapatite is still in progress and apatite is not stoichiometric after 14 days of soaking. A much better developed hydroxyapatite precipitation on Ti-Treat surface will be described in the next section after 28 days of soaking in SBF.

On the other side, the kinetic of apatite formation on the Ti-Treat-Ag sample is different and a shift toward higher zeta potential values can be observed in the basic range of the titration curve of this sample after 14 days of soaking in SBF. A higher content of $\mathrm{Ca}^{++}$ions might explain the shift towards the positive range of the zeta potential measured on this surface: SEM observations, XPS and EDS data [15] show on this sample the presence of a higher amount of hydroxyapatite crystals (with respect to the previous sample) with $\mathrm{Ca}$ and $\mathrm{P}$ in a ratio close to 2.9. Moreover, a deviation of the titration curve towards the positive range is observed at $\mathrm{pH}$ around 8.5: this signal could be related to the desorption of phosphate groups from the non-stoichiometric and unstable hydroxyapatite, even if this hypothesis must be confirmed. Adsorption/desorption phenomena can be recognized on the titration curves because their appearance as a maximum or a minimum [17]. 
It is often reported in literature that presence of a high negative [18] or positive [19] charge on the surface, at physiologic $\mathrm{pH}$, is the critical issue in order to have a bioactive behaviour (hydroxyapatite spontaneous precipitation): zeta potential measurements give a different information. Untreated Ti6Al4V is not bioactive despite a high negative charge at physiologic $\mathrm{pH}$, while bioactive surfaces (Ti-Treat, Ti-Treat-Ag) show a lower negative charge. The key factor in bioactivity and kinetics of apatite precipitation appears to be the presence of specific functional groups and their acidic (or eventual basic) behaviour.

Antibacterial action of the Ti-Treat-Ag sample is related to silver ion release from silver nanoparticles [10]: that is why it is of interest to characterize this surface after soaking in pure water and silver ion release. The alkaline side of the titration curve of a sample after soaking in pure water is reported in the inserted chart of Fig. 1 (called Ti-Treat-Ag_release test). A shift of the alkaline side of the titration curve towards negative values can be detected after soaking in pure water with respect to the Ti-Treat-Ag sample. The shift is small and just a bit higher than the statistical dispersion of the data of the titration curve, but it could be an effect of the complete release of silver ions during soaking in water, in agreement with the XPS and ion release data [15].

\subsection{Functionalization of the Ti6Al4V alloy}

Several natural biomolecules, such as polyphenols, are interesting because of antioxidant, neuroprotective and antitumoral abilities and we made some attempts of coupling them to Ti6Al4V alloy [4]. Functionalization of metal bone implants with biomolecules could allow a local action, overcoming the issue of low bioavailability through systemic administration. In the present research work, gallic acid, as a simple model molecule representing behaviour of natural polyphenols, has been grafted by functionalization to a treated Ti6Al4V substrate in order to improve biological response to bone implants.

The following samples were analysed (Fig. 2): a chemically treated Ti6Al4V sample functionalized with gallic acid (Ti-Treat-Func) tested as prepared and after 28 days of soaking in SBF (Ti-Treat-Func_28 dd SBF). A chemically treated sample tested after 28 days of soaking in SBF is reported in Fig. 2 for a comparison (Ti-Treat_28 dd SBF).

In all these curves, a discontinuity can be observed in the range of $\mathrm{pH}$ between 5.5 and 6: it cannot be explained as a surface reaction at this $\mathrm{pH}$, but it is an artefact due to the apposition of the acid and basic sides of the titration curve which are obtained in different times: if the discontinuity is lower than $10 \mathrm{mV}$ the surface alteration is considered negligible, otherwise different samples should be used for the acid and basic titrations. This discontinuity is not always present and it is not completely senseless: it can be related to surface irreversible reactions which occur in the first side of the titration (often the acid range). For instance, the chemically treated sample (Ti-Treat), already described in Fig. 1 and here reported for a comparison, does not show any discontinuity, revealing reversible surface reactions on the surface during titration.

The titration curve of the Ti-Treat-Func sample shows some distinctive signals: a first small plateau at $\mathrm{pH} 3-4$, a second plateau at $\mathrm{pH}$ 6-7.2 and a maximum around $\mathrm{pH}$ 8.5. On the other side, no relevant shift of the isoelectric point can be observed with respect to the Ti-Treat sample. As a first conclusion, the titration curve gives the information that after functionalization the surface is changed, suggesting a positive result of the process, but the change is compatible with an atomic monolayer and probably not uniform functionalization of the surface, without a coating completely obscuring the substrate (consistent with an unchanged isoelectric point). Moreover, a tentative explanation of the characteristic signals is here reported: some more tests will be performed in order to confirm it. The first plateau 
( $\mathrm{pH} 3-4)$ could be related to ionization of $\mathrm{COOH}$ groups of the gallic acid grafted on the surface according to [12]. The second plateau ( $\mathrm{pH}$ 6-7.2) is likely to be related to the $\mathrm{OH}$ groups of the Ti6Al4V substrate analogously to what observed on the Ti-Treat sample; a contribute from the $\mathrm{OH}$ groups of the grafted acid gallic cannot be excluded. The trend towards positive charge with a maximum around $\mathrm{pH} 8.5$ could be related to decomposition of gallic acid at strong alkaline $\mathrm{pH}$ or to desorption of phosphate groups as already previously observed in the case of unstable hydroxyapatite precipitated on the Ti-Treat-Ag sample after soaking in SBF. XPS data on the Ti-Treat-Func sample [4] show that Ca ions (coming from the SBF solution) are adsorbed during the functionalization process: a small adsorption of phosphate groups may have occurred too.

The curve of titration of the sample Ti-Treat-Func_28 dd SBF is of great interest because SEM observations [15] showed that it is almost completely covered by hydroxyapatite crystals with a $\mathrm{Ca} / \mathrm{P}$ ratio of 1.70 that is comparable with the stoichiometric ratio of stable hydroxyapatite (1.67). The isoelectric point of hydroxyapatite is reported to be around $\mathrm{pH} 5$ [16] and it is known that phosphate groups are preferentially located at the surface of hydroxyapatite giving at physiologic $\mathrm{pH}$ a zeta potential of about $-50 \mathrm{mV}$. These data are in agreement with our results on this sample (isoelectric point at $\mathrm{pH}=4.8$, zeta potential of $-45 \mathrm{mV}$ at $\mathrm{pH}=7.4$ ), while the presence of a plateau in the $\mathrm{pH}$ range between 6-8 is not reported in literature. The exposition of the phosphates and $\mathrm{OH}$ groups of hydroxyapatites could be assumed as an interpretation of this plateau. It must be underlined that stoichiometry of apatite can be strongly influenced by the conditions of precipitation or synthesis, so comparison with literature data is not easy. The abrupt decrease of the curve below $\mathrm{pH} 4.5$ is related to reaction and decomposition of hydroxyapatite [20].

In the case of the sample Ti-treat $28 \mathrm{dd} \mathrm{SBF}$, a titration curve similar to the sample TiTreat-Func_28 dd SBF was detected, confirming the precipitation of a continuous apatite layer, in agreement with SEM, XPS and EDS data [4]. Some differences between the two

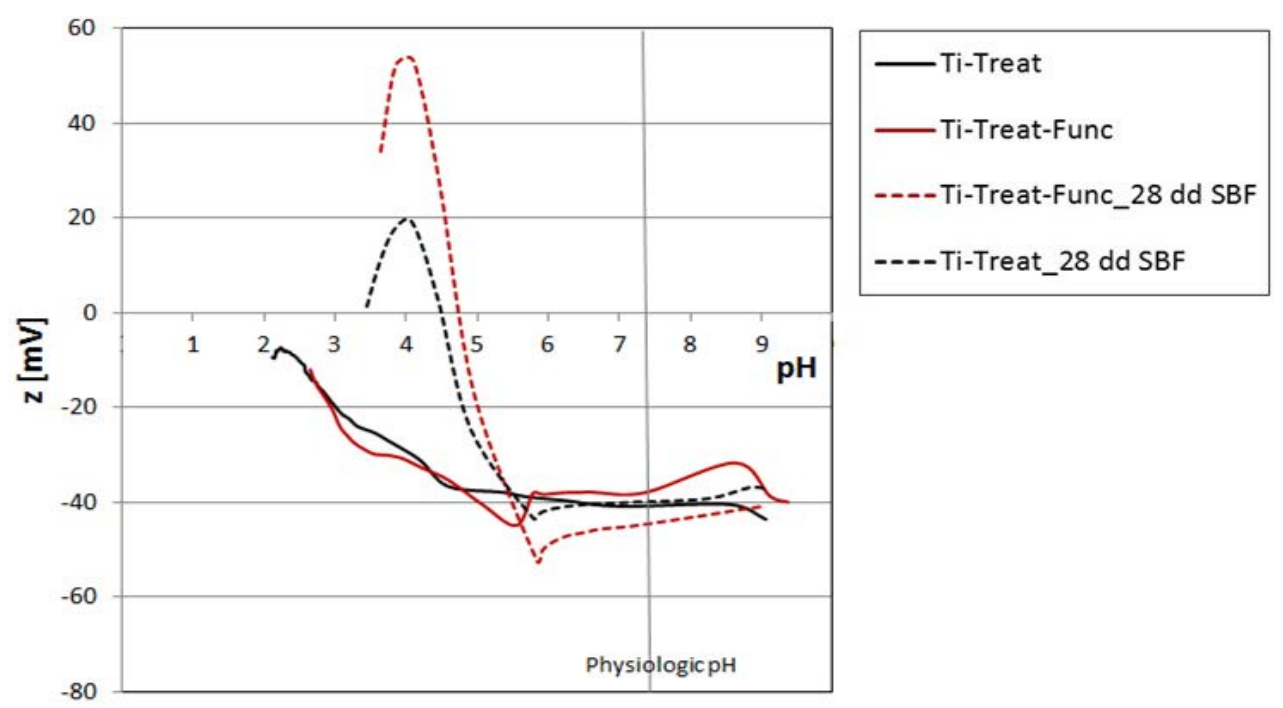

Figure 2: Zeta potential titration vs $\mathrm{pH}$ of the following samples: Ti-Treat, Ti-TreatFunc, Ti-Treat-Func after 15 days of soaking in SBF. 
titration curves can be noted and they could be of interest. As first, the isoelectric point is shifted towards lower $\mathrm{pH}$, then the decomposition of hydroxyapatite at low $\mathrm{pH}$ occurs at a lower surface charge, the plateau in the alkaline range is shifted towards higher surface charge and as last, a maximum appears in the curve at very high $\mathrm{pH}$ values. All these differences could be explained considering precipitation of a less stable hydroxyapatite when gallic acid is not on the surface, but this observation must be further proved.

\subsection{Coating on the Ti6Al4V alloy}

Despite good biocompatibility of $\mathrm{Ti}$ and $\mathrm{Ti}$ alloys, their use in implants is limited sometimes by poor tribological properties which are critical when an implant has mechanical fittings between different parts or in the case of artificial joints. In order to increase fretting, wear and corrosion resistance of Ti6Al4V alloy, a coating of titanium boride was obtained by a thermal treatment in a boron containing salt mixture. Considering that synovial fluid can act as lubricant within an artificial joint and it is mainly constituted by albumin, the adsorption of BSA (Serum Bovine Albumin) on the samples was also tested. The sign and magnitude of zeta potential let us estimate the presence of an adsorbed layer, as well as eventual specific orientation between the solid surface and an adsorbed charged protein. BSA is characterized by the presence of two functional groups (carboxylic and amino groups) which can respectively become negatively and positively charged in water solution depending on $\mathrm{pH}$ : adsorption of the protein usually occurs through electrostatic interactions between the charged functional groups of the protein and the surface.

The zeta potential titration vs $\mathrm{pH}$ was measured on the Ti-coat sample before and after adsorption of BSA, as well as on untreated Ti6Al4V alloy and alumina as reference materials (Fig. 3). Alumina was added as a reference material in this investigation because it currently is a gold standard biomaterial (low wear, low friction coefficient, good lubrication) when tribological properties are considered.

Considering the samples before adsorption, the reference materials (Ti6Al4V untreated alloy and alumina) have an almost linear trend towards negative charge in the alkaline range and they do not show any specific functional group. This is of interest because XPS data on these surfaces show the presence of a not negligible amount of $\mathrm{OH}$ groups [21], but the results from zeta potential measurements evidence they have not a specific acidic/basic behaviour (the degree of amphoteric dissociation of surface hydroxyl groups depends on the properties of the metal ion, such as the charge-to radius ratio and the coordination number [14]). Differently, the titration curve of the titanium boride coating (the Ti-Coat sample) has two evident plateaux corresponding to the presence of respectively strong $(\mathrm{pH}=$ $3.5-4)$ and weak $(\mathrm{pH}=6.5-9)$ acidic $\mathrm{OH}$ groups. This is due to the surface oxidation of titanium boride giving titanium dioxide and boron oxide; the thin layer of titanium oxide on the surface shows a high degree of hydroxylation (as confirmed by XPS analysis) [22], [23]. This result shows that the titanium boride coating is of interest not only for the expected good mechanical and tribological properties, but also for its surface chemistry, because it exposes functional groups with a specific acidic behaviour. As last, all the tested materials before adsorption show a similar negative charge at physiologic $\mathrm{pH}$.

The isoelectric points of the tested materials before adsorption are significantly different (it is at $\mathrm{pH}=3$ for Ti-Coat, at $\mathrm{pH}=4$ for alumina and at $\mathrm{pH}=4.7$ for Ti6Al4V untreated alloy), while the intersection with the horizontal axis becomes the same for all the tested materials after BSA adsorption and it matches the isoelectric point reported in literature for pure BSA $(\mathrm{pH}=4.7-4.9)$ [24]. This result evidences that a continuous adsorbed layer of 
BSA is formed on all the tested materials: at physiological $\mathrm{pH}$ both the protein and the tested surfaces show a negative surface charge, a repulsion effect is expected [25], but zeta potential measurements show that it does not compromise adsorption.

The zeta potential at physiologic $\mathrm{pH}$, after adsorption of albumin, is negative for all the tested materials, but, while the trend of the titration curve of the untreated Ti6Al4V is almost linear, there is an evident plateau in the case of the Ti-Coat ads and Alumina ads samples. Considering that all the surfaces show a similar negative charge before adsorption, a much more similar behaviour with respect to adsorption might have expected. The linear trend is not far from that reported in literature [22] in the case of BSA in solution (random orientation). A possible explanation of the plateaux is that adsorption occurs through electrostatic interaction between the amino group of the protein (even if it is not protonated at physiological $\mathrm{pH}$ ) and the negative $\mathrm{OH}$ groups of the surface; for instance, attraction could occur through the distinctive positive patch on two of the protein surfaces that the protein exhibits at physiological $\mathrm{pH}$ [26]. After adsorption, the carboxylic groups of BSA are exposed on the surface and they are completely ionized at physiologic $\mathrm{pH}$ resulting in a plateau in the alkaline range of the titration curve. Further experiments will be performed in order to clarify why the isoelectric point is eventually not affected by an oriented adsorption (eventually because of a potential dependence of the protein orientation [27]), to quantify the amount of adsorbed BSA molecules on the different surfaces, to evaluate the adsorption bonding strength on the different materials and the influence of the adsorbed layer on the hydrophilicity of the surface. It is also reported [22] that the adsorbed BSA molecules tend to elongate in the direction normal to the contact surfaces with increasing densities of positive surface charge on the substrate and this could have an effect on the zeta potential.

In any case, similarity between the surface charge properties of the titanium boride and alumina samples after BSA adsorption is of interest considering the good lubrication registered on alumina artificial joints.

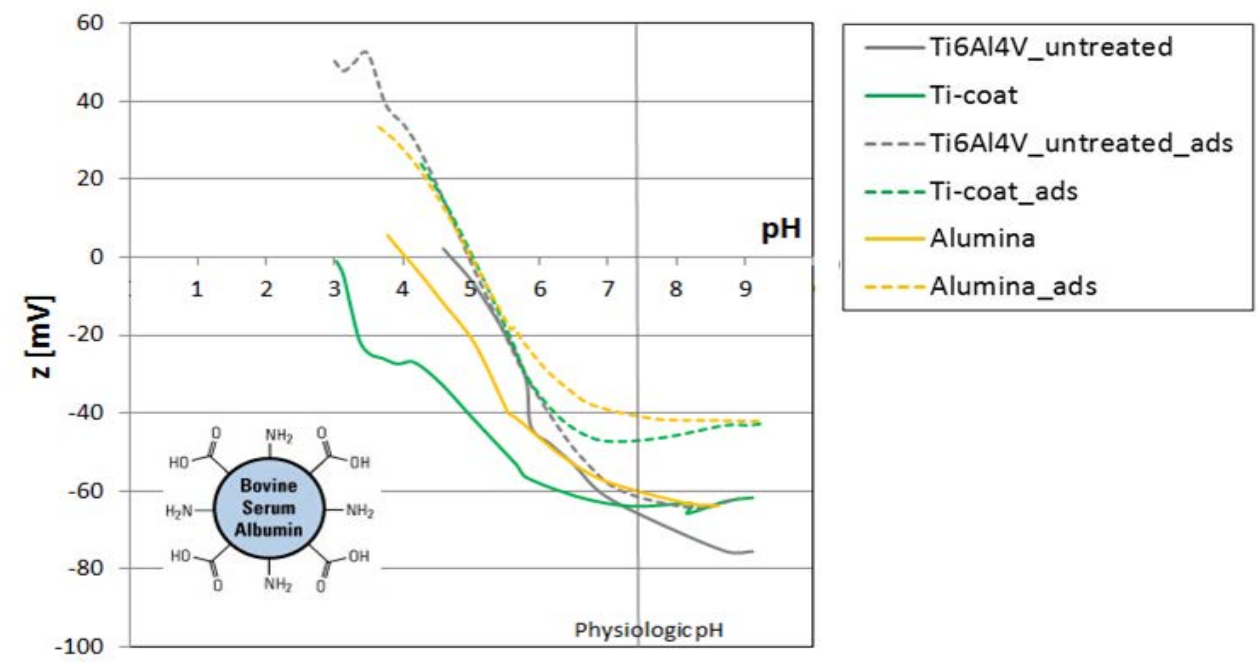

Figure 3: Zeta potential titration vs $\mathrm{pH}$ of the following samples: Ti6Al4V_untreated, TiCoat, Alumina and of the samples Ti6Al4V_untreated_ads, Ti-Coat_ads, Alumina_ads tested after $1 \mathrm{~h}$ of soaking in BSA solution. 


\section{CONCLUSIONS}

Measurements of zeta potential titration vs $\mathrm{pH}$ can give different useful information concerning the surface of biomaterials in complementarity with XPS, EDS, FTIR data and/or SEM observations. The reactivity of the surface of bulk materials vs $\mathrm{pH}$ in aqueous media can be monitored in order to characterize a substrate and to verify the effects on it of chemical treatments, surface modification through functionalization or coatings. Several characteristics of the treated surface can be evidenced such as the eventual presence of an uniform and continuous layer covering the surface, the presence of eventual specific functional groups and their reactivity (protonation or deprotonation vs $\mathrm{pH}$ ), hydrophilic behaviour (making a distinction between topographical and chemical effects), sign and value of surface charge at physiological $\mathrm{pH}$, adsorption of proteins or ions from physiologic fluids, kinetic of hydroxyapatite precipitation and stoichiometry, presence of grafted molecules, occurrence of irreversible surface reaction by modifying $\mathrm{pH}$.

\section{REFERENCES}

[1] Luxbacher, T., The zeta guide principles of the streaming potential technique, Anton Paar, 2014.

[2] Spriano, S., Vernè, E. \& Ferraris, S., Multifunctional Titanium Surfaces for Bone Integration, 2013. European Patent no. 2214732.

[3] Spriano, S., Vernè, E. \& Ferraris, S, Process for producing multifunctional titanium surfaces for reducing the risk of infection and increased bone integration and product made through process. PCT patent pending no. PCT-IT2012-000237.

[4] Cazzola, M., Ferraris, S., Casalegno, V., Prenesti, E. \& Spriano, S., Grafting of gallic acid onto a bioactive Ti6Al4V alloy: a physico-chemical characterization.

[5] Kokubo, T., Bioactive glass ceramics: properties and applications. Biomaterials, 12, pp. 155-163, 1991.

[6] Sarman, B., Tikekar, N.M. \& Ravi Chandran, K.S., Kinetics of growth of super hard boride layers during solid state diffusion of boron into titanium. Ceramics International, 38, pp. 6795-6805, 2012.

[7] Lumetti, S. et al., The response of osteoblastic MC3T3-E1 cells to micro- and nanotextured, hydrophilic and bioactive titanium surfaces. Journal of Materials Science: Materials in Medicine, 27(68), 2016.

[8] Ferraris, S. et al., Surface modification of Ti-6Al-4V alloy for biomineralization and specific biological response: Part I, inorganic modification. Journal of Materials Science: Materials in Medicine, 22(3), pp. 533-545, 2011.

[9] Kokubo, T. \& Takadama, H., How useful is SBF in predicting in vivo bone bioactivity? Biomaterials, 27(15), pp. 2907-2915, 2006.

[10] Ferraris, S., Venturello, A., Miola, M., Cochis, A., Rimondini, L. \& Spriano, S., Antibacterial and bioactive nanostructured titanium surfaces for bone integration. Applied Surface Science, 311, pp. 279-291, 2014.

[11] Roessler, S., Zimmermann, R., Scharnweber, D., Werner, C. \& Worch H., Characterization of oxide layers on Ti6Al4V and titanium by streaming potential and streaming current measurements. Colloids and Surfaces B: Biointerfaces, 26, pp. 387-395, 2002.

[12] Aurousseau, M., Guillet, A. \& Mauret, E, Effect of $p H$ and ionic strength on the electrical charge and particle size distribution of starch nanocrystal suspensions. Starch, 67, pp. 319-327, 2015.

[13] Li, J., Qiao, Y., Ding, Z. \& Liu, X., Microstructure and properties of Ag/N dual ions im-planted titanium. Surface and Coatings Technology, 205, pp. 5430-5436, 2011. 
[14] Takeda, S. \& Fukawa, M., Role of surface OH groups in surface chemical properties of metal oxide films. Materials Science and Engineering: B, 119(3), pp. 265-267, 2005.

[15] Borra V., Master Thesis, Politecnico di Torino, 2014.

[16] Botelho, C.M., Lopes, M.A., Gibson, I.R., Best, S.M. \& Santos, J.D., Structural analysis of Si-substituted hydroxyapatite: zeta potential and X-ray photoelectron spectroscopy. Journal of Materials Science: Materials in Medicine, 13, pp. 11231127, 2002.

[17] Albrecht, T.W.J., Addai-Mensah, J., Fornasiero, D., Effect of pH, Concentration and Temperature on Copper and Zinc Hydroxide Formation/Precipitation in Solution. Chemeca, pp. 18-21, 2011.

[18] Kim,H.M., Himeno, T., Kawashita, M., Lee, J.H., Kokubo, T. \& Nakamura, T., Surface potential change in bioactive titanium metal during the process of apatite formation in simulated body fluid. Journal of Biomedical Materials Research A, 67(4), pp. 1305-1309, 2015.

[19] Kokubo, T. et al., Positively charged bioactive charged bioactive Ti metal prepared by simple chemical and heat treatments. Journal of Royal Society Interface, 7, pp. S503-S513, 2010.

[20] Dorozhkin, S.V., Dissolution mechanism of calcium apatites in acids: A review of literature. Journal of Methodology World, 26, pp. 1-17, 2012.

[21] Spriano, S. et al., How do wettability, zeta potential and hydroxylation degree affect the biological response of biomaterials? Materials Science and Engineering $C$.

[22] Fukuzaki, S., Urano, H. \& Nagata, K., Adsorption of bovine serum albumin onto metal oxide surfaces. Journal of Fermentation and Bioengineering, 81(2), pp. 163167, 1996.

[23] Peretti V., PhD thesis, Politecnico di Torino (in progress).

[24] Hughes Wassell, D.T. \& Embery, G., Adsorption of bovine serum albumin on to titanium powder. Biomaterials, 17, pp. 859-664, 1996.

[25] Cai, K., Frant, M., Bossert, J., Hildebrand, G., Liefeith, K. \& Jandt, K.D., Surface functionalized titanium thin films: Zeta-potential, protein adsorption and cell proliferation. Colloids and Surfaces B: Biointerfaces, 50, pp. 1-8, 2006.

[26] Maffre, P., Brandholt, S., Nienhaus, K., Shang, L., Parak, W.J. \& Beilstein, G.U.N., Effects of surface functionalization on the adsorption of human serum albumin onto nanoparticles - a fluorescence correlation spectroscopy study. Journal of Nanotechnology, 5, pp. 2036-2047, 2014.

[27] Hu, X.N. \& Yang, B.C., Conformation change of bovine serum albumin induced by bioactive titanium metals and its effects on cell behaviour. Journal of Biomedical Materials Research Part A, 102A, pp. 1053-1062, 2014. 